\title{
Genipin cross-linked carbon dots for antimicrobial, bioimaging and bacterial discrimination
}

Chu, Xiaohong; Wu, Fan; Sun, Baohong; Zhang, Ming; Song, Saijie; Zhang, Pan; Wang, Yuli; Zhang, Qicheng; Zhou, Ninglin; Shen, Jian

\section{Published in:}

Colloids and Surfaces B: Biointerfaces

Link to article, DOI:

10.1016/j.colsurfb.2020.110930

Publication date:

2020

Document Version

Peer reviewed version

Link back to DTU Orbit

Citation (APA):

Chu, X., Wu, F., Sun, B., Zhang, M., Song, S., Zhang, P., Wang, Y., Zhang, Q., Zhou, N., \& Shen, J. (2020). Genipin cross-linked carbon dots for antimicrobial, bioimaging and bacterial discrimination. Colloids and Surfaces B: Biointerfaces, 190, [110930]. https://doi.org/10.1016/j.colsurfb.2020.110930

\section{General rights}

Copyright and moral rights for the publications made accessible in the public portal are retained by the authors and/or other copyright owners and it is a condition of accessing publications that users recognise and abide by the legal requirements associated with these rights.

- Users may download and print one copy of any publication from the public portal for the purpose of private study or research.

- You may not further distribute the material or use it for any profit-making activity or commercial gain

- You may freely distribute the URL identifying the publication in the public portal 


\title{
Genipin cross-linked carbon dots for antimicrobial, bioimaging and bacterial discrimination
}

Xiaohong Chu, ${ }^{a, b}$ Fan Wu, ${ }^{a, b}$ Baohong Sun, ${ }^{a, b}$ Ming Zhang, ${ }^{\text {a,d }}$ Saijie Song, ${ }^{a, b}$ Pan Zhang, ${ }^{\text {a,b }}$ Yuli Wang, ${ }^{\text {a,b }}$ Qicheng Zhang, ${ }^{\text {a,b }}$ Ninglin Zhou, ${ }^{a, b, c *}$ Jian Shen ${ }^{a, b *}$

a Jiangsu Collaborative Innovation Center for biological functional materials, College of Chemistry and Materials Science, Nanjing Normal University, Nanjing 210023, China;

${ }^{b}$ Jiangsu Key Laboratory of Biofunctional Materials, Jiangsu Engineering Research Center for Biomedical Function Materials, Nanjing 210023, China;

${ }^{c}$ Nanjing Zhou Ninglin Advanced Materials Technology Company Limited, Nanjing 211505, China;

${ }^{d}$ Department of Health Technology, Technical University of Denmark, Kongens Lyngby, 2800, Denmark;

E-mail addresses: zhouninglin@njnu.edu.cn (N. Zhou), jshen@njnu.edu.cn (J. Shen).

\begin{abstract}
Multifunctional carbon dots (CDs) present enormous potential in numerous applications and have attracted widespread attention for various applications in the biomedical field. Bacterial infection is a common health issue; the development of antibacterial materials with low toxicity and good biocompatibility is becoming more important. In this work, we synthesized a new type of nitrogen co-doped carbon dots-genipin covalent conjugate (N-CDs-GP) via hydrothermal methods. The microstructure and chemical composition of the N-CDs-GP were characterized. The biocompatibility, stability, antibacterial activity, and fluorescence performance of the N-CDs-GP were assessed. The results revealed that N-CDs-GP possessed high biocompatibility, high light stability, and broad antibacterial activity. Additionally, selective Gram-positive bacterial imaging by N-CDs-GP provided a more rapid
\end{abstract}


method of bacterial detection. The N-CDs-GP have the potential to be applied as bioimaging and antibacterial agents and for bacterial discrimination.

Keywords: multifunctional carbon dots; antibacterial activity; bioimaging; bacterial discrimination

\section{Introduction}

Bacterial infection has become one of the largest global threats to public health; if not addressed, these infections lead to morbidity and mortality [1-3]. Conventionally, the most extensively used method of treating bacterial infection is antibiotic therapy [4-6]. However, the over abuse of antibiotics has resulted in low efficacy due to developed resistances [7]. As a result, conventional antibiotics lose their ability to prevent bacterial growth under these circumstances [8]. Therefore, efforts have been devoted to exploring alternative methods for treating bacterial infection. Nanotechnology has been recognized as a new type of antibacterial therapy and has shown promising efficacy. The antibacterial activity of nanoparticles (NPs) is due to their small size, allowing the penetration and compromising of cell membranes [9, 10$]$. Therefore, nanomaterials could be effective antibacterial agents and include silver (Ag) [11], titanium oxide $\left(\mathrm{TiO}_{2}\right)$ [12], copper oxide $(\mathrm{CuO})$ [13] and zinc oxide $(\mathrm{ZnO})$ [14]. However, these nanomaterials may have limited clinical applications because of their cytotoxicity or prolonged retention. Accordingly, studies have sought to design materials with low toxicity and good biocompatibility while possessing high antibacterial activity.

Carbon nanomaterials are highly appealing for applications in bioimaging, optoelectronics, and photocatalysis [15-21]. Carbon dots (CDs), one family of quantum dot-like carbon nanomaterials, are easily modified and have low cytotoxicity, high light stability, and good aqueous solubility [22, 23]. Due to their outstanding physicochemical properties, CDs are promising nanomaterials for biomedical applications and have been used in drug and gene delivery systems [24-26]. These nanoscale CDs also have potential as bactericides [27-31]. Combined antibacterial and bioimaging applications of $\mathrm{CDs}$ have unique advantages over traditional fungicides. CDs are easily modified with specific functional groups for various 
applications, including drug delivery, sensors, bioimaging, and bacterial labeling. Li et al. synthesized bright yellow fluorescent CDs for the label-free detection of fluoroquinolone and histidine [32]. Liu et al. used a simple microwave method to prepare CDs as a vaccine delivery system for intranasal immunization wherein ovalbumin antigen was delivered using CDs as an antigen carrier [33]. Yang et al. used the antibiotic metronidazole to prepare CDs via hydrothermal methods [34]. These synthesized CDs were used to simultaneously fluorescently label and kill obligate anaerobes but were less effective due to drug-resistant bacterial strains. These findings indicate that CDs can be applied in various fields but require further work for their simultaneous application in different fields. Therefore, it is desirable to develop an antimicrobial material with low toxicity and good biocompatibility and can be applied in bioimaging. As such, we synthesized a novel nitrogen co-doped carbon dots-genipin covalent conjugate (N-CDs-GP) by conjugating $\mathrm{N}-\mathrm{CDs}$ with genipin (GP). L-Tryptophan and chlorhexidine acetate (CHA) were used as carbon sources, and GP was selected as a cross-linking agent to synthesize multifunctional nanoparticles. CHA, as a biguanide antiseptic and disinfectant, has been widely used in medical applications [35]. CHA was chosen as one of the carbon sources due to its broad-spectrum antimicrobial activity [36-38]. GP, extracted from gardenia fruits, is an excellent natural bio-crosslinker and is widely used in herbal medicines and food dyes. GP is considered an ideal replacement for other crosslinkers because GP forms stable crosslinked networks and shows higher compatibility and lower cytotoxicity than other crosslinkers, such as formaldehyde, glutaraldehyde, and epoxy [39-41]. GP is also used as anti-inflammatory and choleretic drugs [42-45]. CHA and L-tryptophan were conjugated via carboxyl-amine reactions. GP reacts with amine groups of N-CDs, producing green-colored fluorescent particles (Fig. 1). The N-CDs-GP has superior biocompatibility, lower cytotoxicity, and better antibacterial properties than N-CDs. Furthermore, N-CDs-GP was prepared by a simple method and has antibacterial and bioimaging applications. In vitro and in vivo tests demonstrated N-CDs-GP has potential to treat local tissue infections. This study proposed multifunctional, fluorescent, cross-linked carbon dots for simultaneously inhibiting 
bacterial growth, selectively imaging Gram-positive bacteria, and accelerating wound repair.
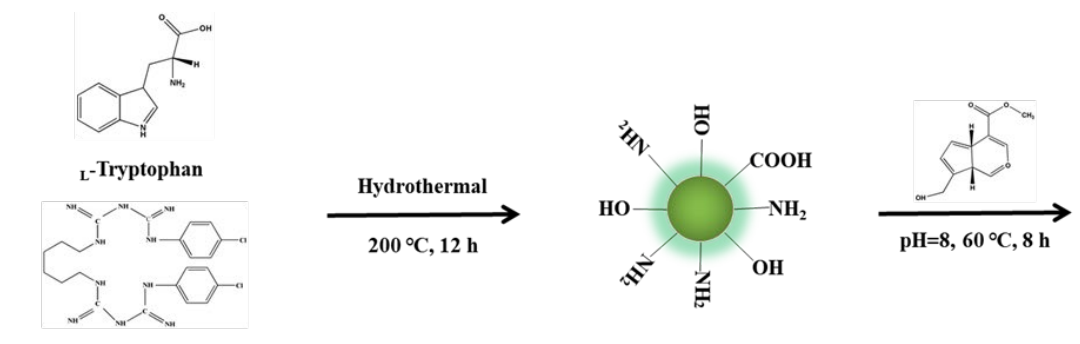

Chlorhexidine Acetate
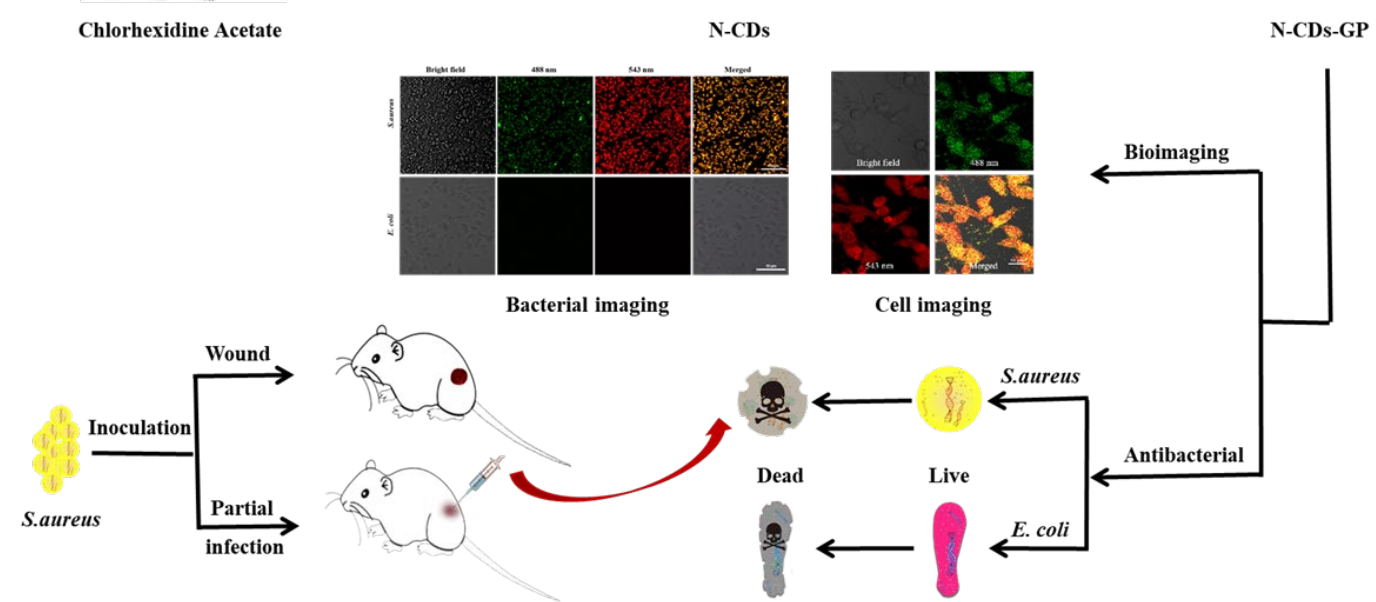

Fig. 1 Schematic diagram of the synthesis and application of N-CDs and N-CDs-GP.

\section{Experimental Section}

\subsection{Materials}

L-tryptophan and chlorhexidine acetate were purchased from Shanghai Huixing Biochemical Reagent Co., Ltd. and Shanghai Macklin Biochemical Co., Ltd., respectively. Genipin was obtained from Chengdu Kangbang Biological Technology Co., Ltd. All of the reagents were analytical reagent and deionized water (18.2 $\mathrm{M} \Omega$ $\mathrm{cm})$ was supplied for all experiments.

Staphylococcus aureus (S. aureus, ATCC 25923) and Escherichia coli (E.coli, ATCC 25922) strains were obtained by the Jiangsu Provincial Center for Disease Control and prevention. Fresh whole blood and human breast cancer cell were separately purchased from Jiangsu blood center and the Cell Bank of the Culture Collection of the Chinese Academy of Sciences (Shanghai). Fetal bovine serum (FBS) and Dulbecco's modified eagle medium (DMEM) were purchased from SunShine Biotechnology Co., Ltd. The remaining biological reagents were from Hyclone Co., Ltd., such as penicillin, phosphate-buffered saline (PBS), streptomycin, and methyl 
thiazolyl tetrazolium (MTT). Animal experiments were performed according to the care and use guidelines for experimental animals. All experiments were conducted following the system guidance of Nanjing Normal University.

\subsection{Characterization}

The morphologies of the samples were observed using a Tecnai G2 F20 (FEI, US) transmission electron microscope (TEM). The $\zeta$-Potential and dynamic light scattering (DLS) analysis were performed with Zetasizer Nano ZS90 (Malvern, UK) nanoparticle size potential analyzer. The X-ray diffraction (XRD) analysis was performed using a D8-ADVANCE X-ray diffractometer (BRUKER, Germany). The TGA measurements were performed on a STA449F3 (NETZSCH, Germany) thermogravimetric/differential thermal integrated thermal analyzer. UV-vis absorption spectra and photoluminescence (PL) spectra were recorded using Cary 50 (Agilent, Australia) and F-7000F-7000 (Hitachi, Japan) spectrophotometer, respectively. The functional groups of the samples were determined by a Nexus 670 (Thermo Nicolet, US) FTIR thermal spectrophotometer. Meanwhile, the chemical composition and elemental analysis of the samples were recorded using EscaLab 250Xi (Thermo Fisher Scientific, US) X-ray photoelectron spectroscopy (XPS). Finally, the fluorescent photos of cells were acquired by TI-E-A1R (Nikon, Japan) confocal laser scanning microscopy (CLSM). The determination of the quantum yields (QYs) of N-CDs-GP was similar to a previous report [26].

\section{Results and discussion}

\subsection{Preparation and characterization of $\mathrm{N}-\mathrm{CDs}-\mathrm{GP}$}

N-CDs-GP particles were prepared through a one-step hydrothermal method using L-tryptophan and CHA as shown in Fig. 1. Initially, L-tryptophan and CHA underwent amidation polycondensation. The obtained peptide chain was then carbonized under extended reaction time. GP was used as a cross-linking agent to improve biocompatible and antibacterial properties. TEM images show that $\mathrm{N}-\mathrm{CDs}$ and N-CDs-GP were spherical nanoparticles with average sizes of $\sim 4.0 \mathrm{~nm}$ and $\sim 80.0 \mathrm{~nm}$, respectively (Fig. 2A and B). N-CDs formed aggregates after GP cross-linking. The high-resolution TEM images in Fig. S1A and B show that the crystal lattice spacing of 
N-CDs and N-CDs-GP were 0.32 and $0.31 \mathrm{~nm}$, corresponding to the (002) crystal plane diffraction peak, which indicated the presence of amorphous carbon. Crosslinking with GP did not alter the structure of the N-CDs. DLS results showed that the average hydrodynamic diameters of N-CDs and N-CDs-GP were 5.2 and 90.0 $\mathrm{nm}$, respectively (Fig. S2A and B). The hydrodynamic diameters of the two materials were consistent with the dimensions measured by TEM (DLS measures overall hydrodynamic size). XPS scans of N-CDs-GP indicated the particles comprised oxygen, nitrogen, and carbon with binding energy peaks at 532.55, 399.49, and $284.82 \mathrm{eV}$ corresponding to O1s, N1s, and C1s, respectively (Fig. 3A). Fig. S3(A-C) show corresponding high-resolution XPS images of $\mathrm{C} 1 \mathrm{~s}, \mathrm{~N} 1 \mathrm{~s}$, and $\mathrm{O} 1 \mathrm{~s}$, respectively. The three C1s peaks centered at 284.6, 285.58, and $287.7 \mathrm{eV}$ were ascribed to $\mathrm{C}-\mathrm{C} / \mathrm{C}=\mathrm{C}, \mathrm{C}-\mathrm{N}$, and $\mathrm{C}=\mathrm{N} / \mathrm{C}=\mathrm{O}$ groups, respectively. The high-resolution $\mathrm{N} 1 \mathrm{~s}$ spectrum was deconvoluted into three peaks at 399.2, 399.9, and $401.6 \mathrm{eV}$, corresponding to $\mathrm{C}-\mathrm{N}-\mathrm{C}, \mathrm{N}-(\mathrm{C})_{3}$, and $\mathrm{N}-\mathrm{H}$, respectively. For $\mathrm{O} 1 \mathrm{~s}$, three fitting peaks at $531.65,532.4$, and $533.1 \mathrm{eV}$ corresponding to the $\mathrm{C}=\mathrm{O}, \mathrm{C}-\mathrm{OH}$, and $\mathrm{C}-\mathrm{O}-\mathrm{C}$ bonds, respectively, were observed. The typical XRD patterns of N-CDs and N-CDs-GP in Fig. S4 showed broad reflection peaks at $2 \theta=27.84^{\circ}$ and $2 \theta=28.90^{\circ}$, respectively. The interlayer spacing of N-CDs and N-CDs-GP were calculated as $0.32 \mathrm{~nm}$ and 0.31 $\mathrm{nm}$ (according to $2 d \sin \theta=\mathrm{n} \lambda$, where $d$ is the layer spacing, and $\lambda$ is approximately $0.154 \mathrm{~nm}$ ), which were similar to bulk graphite, displaying good crystallization properties. These results were consistent with the high-resolution TEM analyses. The surface structures of N-CDs, GP, and N-CDs-GP were characterized by FTIR spectroscopy. N-CDs-GP had nearly similar characteristic peaks as N-CDs and GP (Fig. 3B). A strong broad peak of N-CDs-GP at $3400 \mathrm{~cm}^{-1}$ indicated the presence of $\mathrm{O}-\mathrm{H}$ or N-H tensile vibration. Absorption bands at 2940, 1660, 1300, and $1100 \mathrm{~cm}^{-1}$ corresponded with stretching modes of $\mathrm{C}-\mathrm{H}, \mathrm{C}=\mathrm{O}, \mathrm{C}-\mathrm{N}$ and $\mathrm{C}-\mathrm{O}$, respectively, which indicated high densities of hydroxyl or amine groups on N-CDs-GP surfaces. These results indicated N-CDs-GP was successfully synthesized. The thermostabilities of $\mathrm{N}-\mathrm{CDs}$ and N-CDs-GP were investigated by TGA in a nitrogen atmosphere. Fig. S5 shows that N-CDs started losing mass at $52{ }^{\circ} \mathrm{C}$, whereas N-CDs-GP began to lose 
mass at $130{ }^{\circ} \mathrm{C}$. This indicated that N-CDs-GP had increased thermostability compared with N-CDs. Further, the $\zeta$ potential of N-CDs changed from -8.82 to -17.3 $\mathrm{mV}$ after GP cross-linking, which could be attributed to GP reactions with N-CDs amino groups (Fig. S6A and B).

A
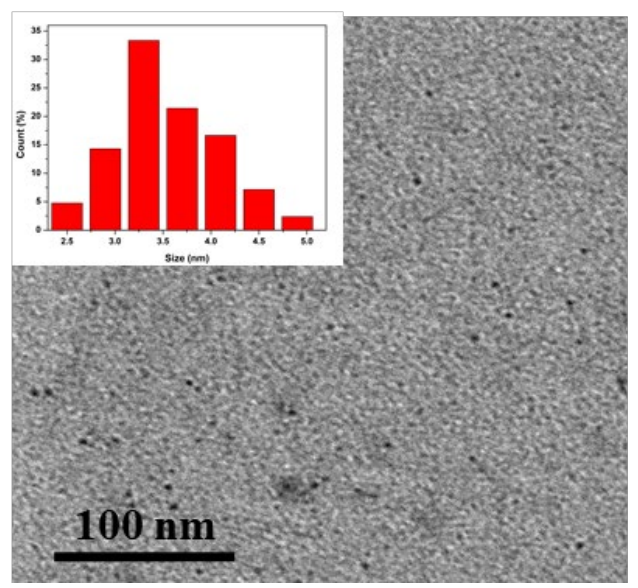

$\mathrm{B}$

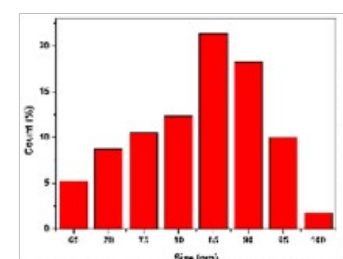

$100 \mathrm{~nm}$

Fig. 2 TEM images and size distribution (inset) of N-CDs (A) and N-CDs-GP (B).
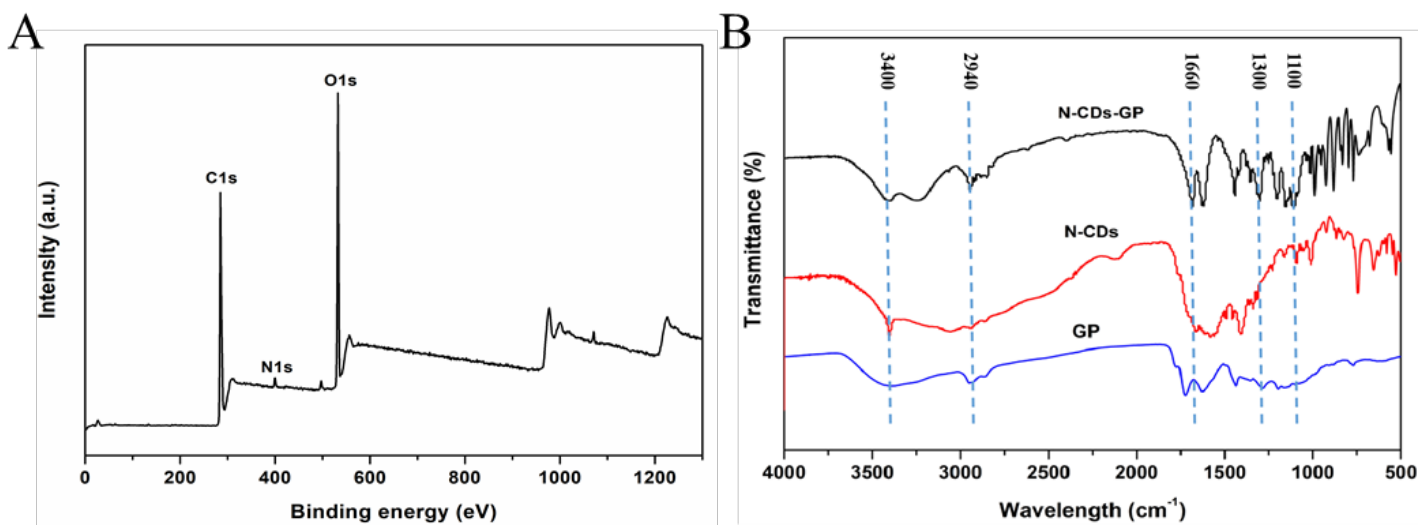

Fig. 3 (A) XPS of N-CDs-GP. (B) FTIR spectra of N-CDs, GP, and N-CDs-GP.

\subsection{Spectral characteristics}

Optical properties were measured using PL and UV-vis spectroscopy. As shown in Fig. 4A, the excitation ( $\lambda$ ex) peaks of N-CDs and N-CDs-GP are at 360 and $380 \mathrm{~nm}$, and the emission ( $\lambda \mathrm{em})$ peaks are at 450 and $470 \mathrm{~nm}$, respectively. The N-CDs and N-CDs-GP solutions exhibited different green luminescence under $365 \mathrm{~nm}$ UV lamp (Fig. 4A, inset). Fig. S7 shows that the PL emission spectra of N-CDs redshifted from 390 to $460 \mathrm{~nm}$ as the excitation wavelength increased from 310 to $390 \mathrm{~nm}$, whereas the PL emission peak of N-CDs-GP redshifted from 470 to $490 \mathrm{~nm}$ as the excitation 
wavelength increased from 300 to $420 \mathrm{~nm}$ (Fig. S8). The maximum PL intensities of N-CDs and N-CDs-GP were obtained at 428 and $473 \mathrm{~nm}$ under 350 and $380 \mathrm{~nm}$ excitation wavelengths. The spectra showed typical excitation wavelength dependence. Using quinine sulfate as a reference, $\left(\mathrm{QY}=54 \%\right.$ in $\left.0.1 \mathrm{M} \mathrm{H}_{2} \mathrm{SO}_{4}\right)$, the photoluminescence quantum yield of N-CDs-GP was calculated to be $4.7 \%$. To investigate the applicability of N-CDs-GP as a fluorescent biomarker in physiological environments, the fluorescent stability of $\mathrm{N}-\mathrm{CDs}-\mathrm{GP}$ aqueous solutions at various $\mathrm{pH}$ values was evaluated. As shown in Fig. S9, while the emission wavelength of $\mathrm{N}-\mathrm{CDs}-\mathrm{GP}$ was constant, the fluorescence intensity was affected. At $\mathrm{pH}$ values of 1 and 13, the fluorescence intensity of N-CDs-GP was nearly quenched; as the $\mathrm{pH}$ increased from 3 to 11, the fluorescence intensity of N-CDs-GP generally decreased. The results indicated that N-CDs-GP has potential applications in bioimaging. N-CDs and N-CDs-GP were thoroughly dispersed in distilled water for UV-vis analysis. The characteristic peaks of GP and N-CDs at approximately 240 and $290 \mathrm{~nm}$, respectively, were also observed for N-CDs-GP (Fig. 4B). These peaks are likely attributed to $\pi-\pi^{*}$ electronic transitions for carbon nitrides.
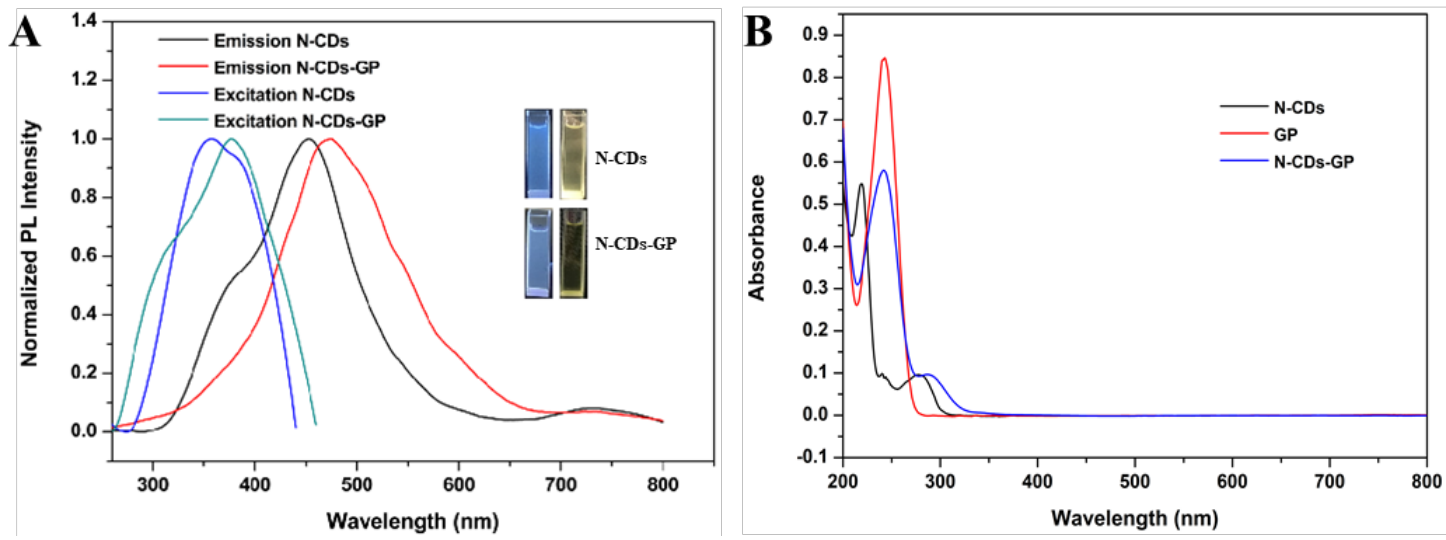

Fig. 4 (A) Normalized photoluminescence excitation and emission spectra of N-CDs and N-CDs-GP dispersed in water. Insets show images of N-CDs and N-CDs-GP aqueous solutions under ambient light and $365 \mathrm{~nm}$ UV lamp. (B) UV-vis absorption spectra of N-CDs, GP, and N-CDs-GP.

\subsection{Cell imaging and cytotoxicity of $N-C D s-G P$}

The cell imaging ability of N-CDs-GP were explored using MCF-7 cells. Fig. 5A 
shows the cells exhibited bright green and red fluorescence when excited with 488 and $543 \mathrm{~nm}$ laser pulses using CLSM, respectively. N-CDs-GP had good photostability and low photobleaching. The emission of polychromatic fluorescence without any discernable morphological defects demonstrated that N-CDs-GP had excellent biocompatibility. In determining the suitability of a compound as a bactericide in living organisms, it is necessary to evaluate the safety of the compound. MTT and hemolysis experiments were carried out to evaluate the biocompatibility of N-CDs and N-CDs-GP. Toxicity is an important factor limiting the biological applications of nanomaterials. To broaden nanomaterial applications in biomedicine, it is essential to evaluate their biological toxicity [46]. The in vitro cytotoxicity of N-CDs, GP, and N-CDs-GP were studied using MCF-7 cells by standard MTT. Fig. S10A and B showed the proliferation of cells co-cultured with different concentrations of N-CDs, GP, and N-CDs-GP for 24 and $48 \mathrm{~h}$, respectively. The results showed that the cell proliferation rate tended to decrease with increasing concentration but remained above $85 \%$. As the culture time increased, no significant changes in proliferation rate were observed. These results indicated that N-CDs-GP has great potential as a low-toxicity antibacterial agent [47]. Next, the hemolytic activity of N-CDs and N-CDs-GP were examined. Red blood cells (RBCs) in PBS and distilled water were used as negative and positive controls, respectively. Hemolysis refers to the interaction and incompatibility of samples with RBCs. When the hemolysis rate of a material is less than $5 \%$, it is recognized as biologically safe $[48,49]$. As shown in Fig. S11, the highest hemolysis rates of N-CDs and N-CDs-GP were approximately $4.2 \%$ and $3.6 \%$, which were lower than the threshold level. Fig. 5B shows that RBCs treated with N-CDs-GP exhibited typical morphological biconcave shapes compared with negative control; RBCs treated with high concentrations of N-CDs exhibited roughly normal morphological biconcave shapes. These results were consistent with those of the hemolysis analysis, suggesting that N-CDs-GP had better biocompatibility than N-CDs. Thus, N-CDs-GP can act as a potential candidate for multicolor cell imaging applications and as a potential antibacterial agent with high efficacy. 

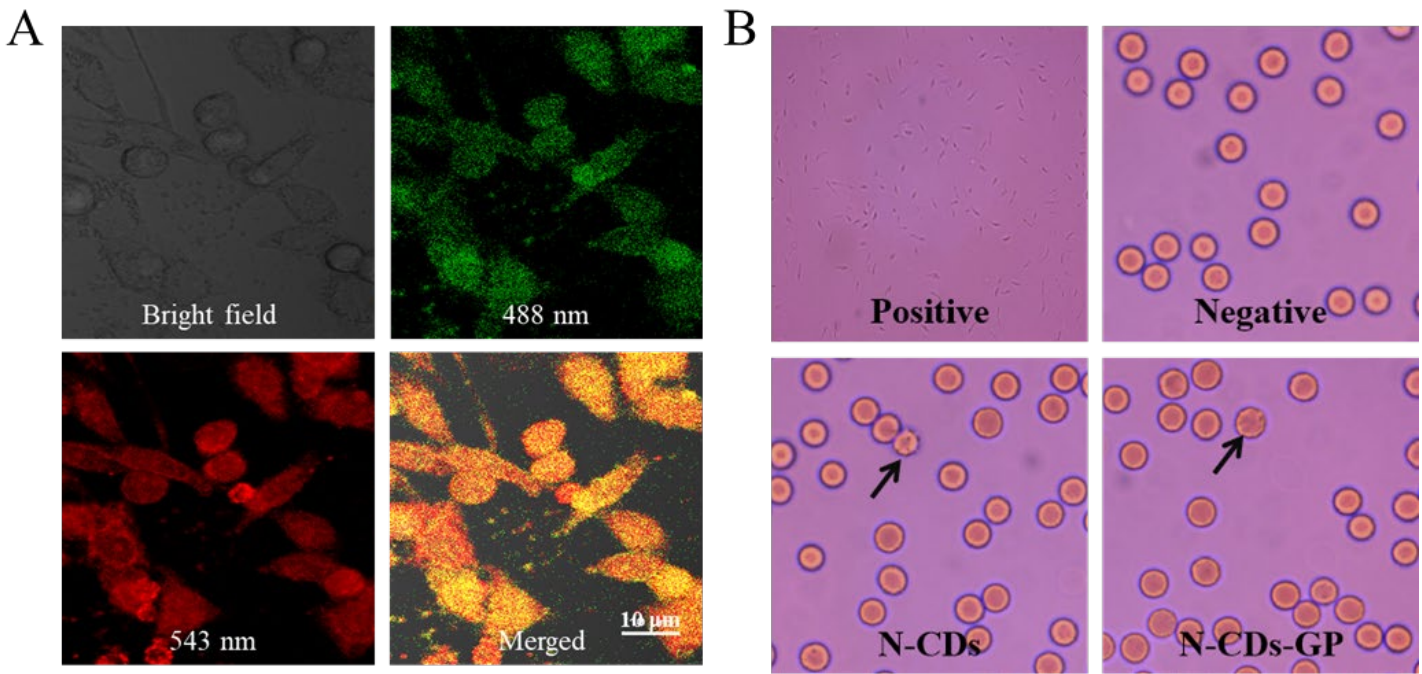

Fig. 5 Cell imaging and cytotoxicity of the synthesized compound. (A) CLSM images of MCF-7 cells cultured with N-CDs-GP observed under bright-field, $488 \mathrm{~nm}, 543 \mathrm{~nm}$, and their merged images, respectively. (B) The optical images of RBCs from positive control, negative control, $1.0 \mathrm{mg} / \mathrm{mL}$ of the N-CDs, and $1.0 \mathrm{mg} / \mathrm{mL}$ of the N-CDs-GP.

\subsection{Selective CLSM imaging of Gram-positive bacteria}

Given the super fluorescence performance of N-CDs-GP, bacterial imaging can be realized [50, 51]. Gram-positive $S$. aureus and Gram-negative E. coli bacteria were selected to assess the imaging performance of N-CDs-GP by confocal microscopy. As displayed in Fig. 6, multi-color fluorescence signals from N-CDs-GP were visualized under different excitation wavelengths for $S$. aureus after incubating with N-CDs-GP for $1 \mathrm{~h}$. However, E. coli cells showed no apparent fluorescent signals under the same conditions. To further verify the selectivity of N-CDs-GP for S. aureus, the zeta potential of both bacteria were measured with and without treatment with N-CDs-GP. Fig. S12 showed the $\zeta$ potential of $S$. aureus changed from -15.7 to $-28.3 \mathrm{mV}$ and that of $E$. coli changed from -16.5 to $-18.2 \mathrm{mV}$. The results indicated that interactions between $S$. aureus and N-CDs-GP were primarily dependent on sectional interactions between the negatively charged bacteria surface and the positively protonated amide group of the N-CDs-GP; the zeta potential of the N-CDs-GP may have had little effect to the interaction [1]. The ability of N-CDs-GP to selectively image Gram-positive bacteria may be caused by the differences in cell surface structure and composition between the two types of bacteria. Generally, Gram-positive bacteria have thick but 
structurally simple cell walls similar to network structures comprising a peptidoglycan layer interconnected with the plasma membrane $[52,53]$. A number of anionic sites provided by teichoic acid on the peptidoglycan layer promote the electrostatic binding of materials to the bacteria surface. The cell walls of Gram-negative bacteria consist of a thin layer of glycan between the protective extracellular membrane and the cytoplasmic membrane, and the outer membrane is enriched with lipopolysaccharides, which are cross-bridged with divalent cations [54-56]. Due to the unique structure and composition of the outer membrane, nanoparticles or hydrophobic molecules have difficulty interacting with Gram-negative bacteria. Our results indicated that N-CDs-GP can interact more effectively with Gram-positive bacteria than Gram-negative bacteria.
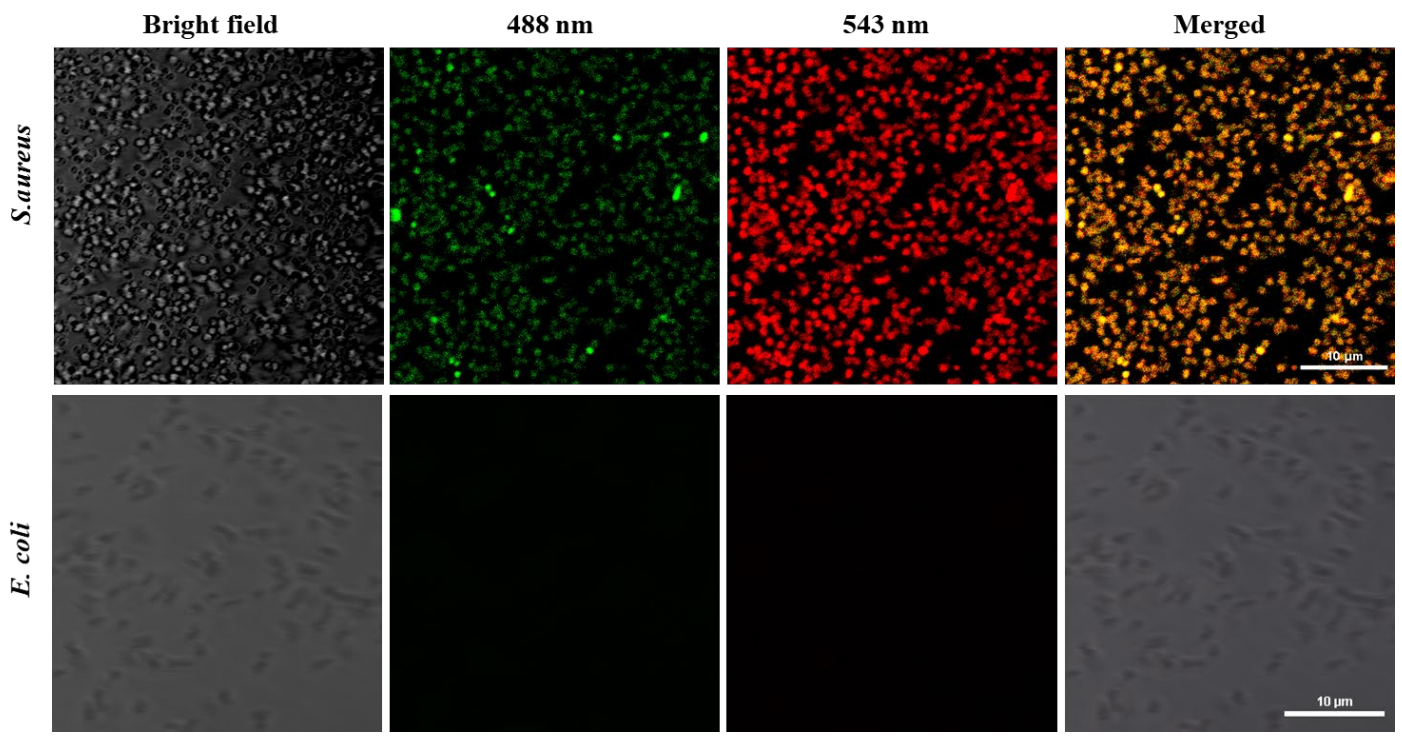

Fig. 6 CLSM images of $S$. aureus and E. coli cultured with N-CDs-GP for $1 \mathrm{~h}$ observed under bright-field, $488 \mathrm{~nm}, 543 \mathrm{~nm}$, and their merged images.

\subsection{In vitro antibacterial activity of $N-C D s-G P$}

The antibacterial abilities of N-CDs and N-CDs-GP against E. coli and S. aureus were evaluated using the minimum inhibitory concentration (MIC) method. To explore the concentration effects of N-CDs and N-CDs-GP on antibacterial activity, a series of different doses of N-CDs and N-CDs-GP were incubated with the two types of bacteria, respectively. The O.D. values of the mixtures were measured at $595 \mathrm{~nm}$ (Fig. S13A and B). The MIC values of the two types of bacteria were 1.0 and 2.0 
$\mathrm{mg} / \mathrm{mL}$, respectively. N-CDs-GP had a better antibacterial activity compared with N-CDs. Bacterial inhibition was also measured by MTT assay after incubating N-CDs and N-CDs-GP with the two bacteria, respectively. The O.D. values of the mixtures were measured at $510 \mathrm{~nm}$. Fig. S14A and B indicate that N-CDs-GP had a better inhibitory effect against bacteria compared with N-CDs. Colony-forming unit (CFU) counting means were performed to determine the antibacterial activity of N-CDs and N-CDs-GP. Fig. 7A shows that the density of bacterial growth significantly decreased with increasing concentrations of N-CDs and N-CDs-GP. Interestingly, N-CDs-GP had a stronger antibacterial effect compared with N-CDs. These results suggested that the crosslinking of GP could improve the antibacterial ability of N-CDs.

To further study the anti-bacterial effects of N-CDs-GP, morphological changes in bacteria were investigated by SEM. As shown in Fig. 7B, untreated bacteria cell membranes had clear edges and surface integrity. After treating with N-CDs-GP, the outer membranes of both bacteria collapsed and intracellular content leakage were observed. The synthesized N-CDs contains amide groups; when partially protonated, a positive charge is obtained [57-59]. GP cross-linking aggregates a large number of carbon dots together yielding stronger antibacterial ability. The as-prepared N-CDs-GP can interact with the negatively charged lipid membrane through electrostatic and hydrophobic reactions and induce damage to bacteria cell walls, leading to intracellular electrolyte leakage and cell death. 

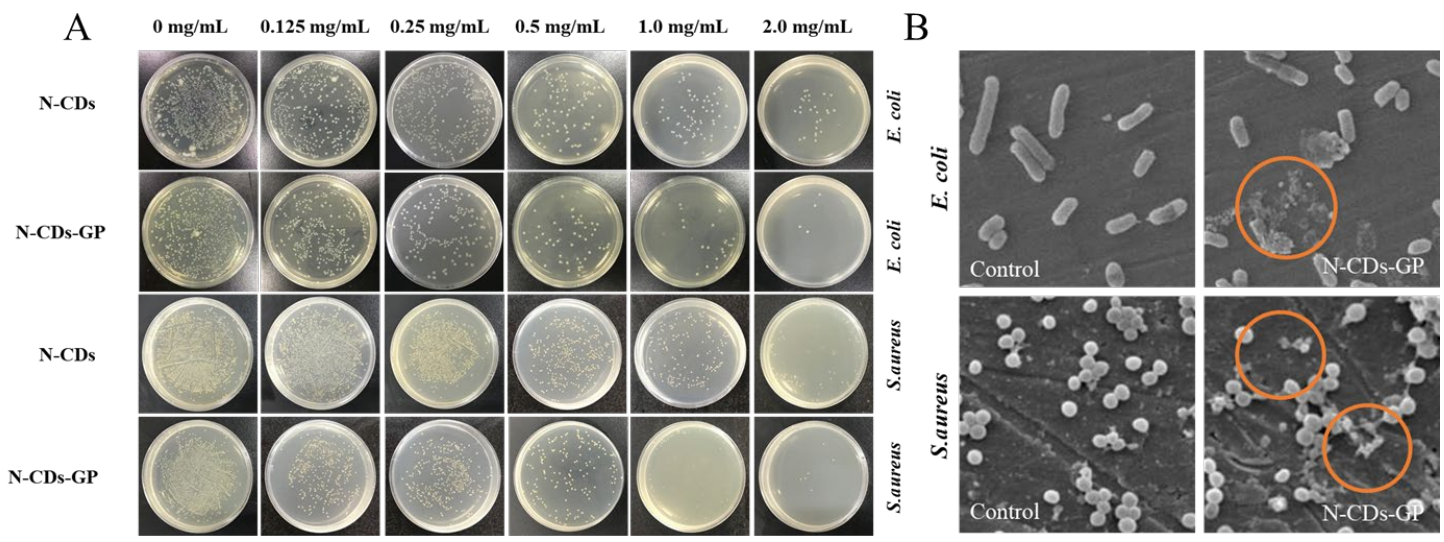

Fig. 7 (A) Colony status images of different doses of N-CDs and N-CDs-GP with liquid media containing the same amounts of E. coli and S. aureus. (B) SEM images of $E$. coli and $S$. aureus after incubating $6 \mathrm{~h}$ at $37^{\circ} \mathrm{C}$ (untreated E. coli; N-CDs-GP treated E. coli; untreated S. aureus; N-CDs-GP treated S. aureus).

\subsection{In vivo antibacterial activity of $N-C D s-G P$}

To further investigate the in vivo antibacterial property of N-CDs-GP, mice infected with $S$. aureus were established as models. In this work, colony number and wound healing observations were recorded to explore the antibacterial efficacy of N-CDs-GP. For the colony number experiment, the mice were divided into a control group and an experimental group (Fig. 8A). The antibacterial efficacy was assessed by comparing the bacterial counts in the infected site. Fig. S15 shows that the bacteria count in the infected tissue after treatment was much lower than that in the control group. These results indicated that N-CDs-GP possessed excellent antibacterial properties.

To evaluate the therapeutic effects of N-CDs-GP on infected wounds, mice models were divided into a control group and an experimental group. Fig. 8B presents images of the wound healing process at different time points. Fresh postoperative wounds with diameters of approximately $1.2 \mathrm{~cm}$ were infected with $S$. aureus. Two days after infection, abscesses appeared on the surfaces of the infected wounds and were treated with either PBS or N-CDs-GP. After 4 days, scabs appeared on infected wounds treated with N-CDs-GP, whereas abscesses were observed on the control wounds. After 7 days, the wound areas of the N-CDs-GP treatment group were significantly smaller than those of the control group. After 14 days, the wound areas of each group 
were significantly reduced; the wounds treated with N-CDs-GP had healed, and the control group had not completely healed. The wound areas in the control and N-CDs-GP groups were $12.5 \%$ and $0.5 \%$ of the original wound areas, respectively. These results suggested that N-CDs-GP had excellent antibacterial activity.

The in vivo toxicity of N-CDs-GP was also tested. N-CDs-GP solution was injected once a day into the infected sites of mice. After 3 days of treatment with PBS or N-CDs-GP, the mice were sacrificed, and the main organs were collected and subjected to H\&E staining. Fig. S16 shows the tissues of the mice treated with N-CDs-GP were not damaged compared with the control group. The results demonstrated that the N-CDs-GP had good in vivo biosafety.

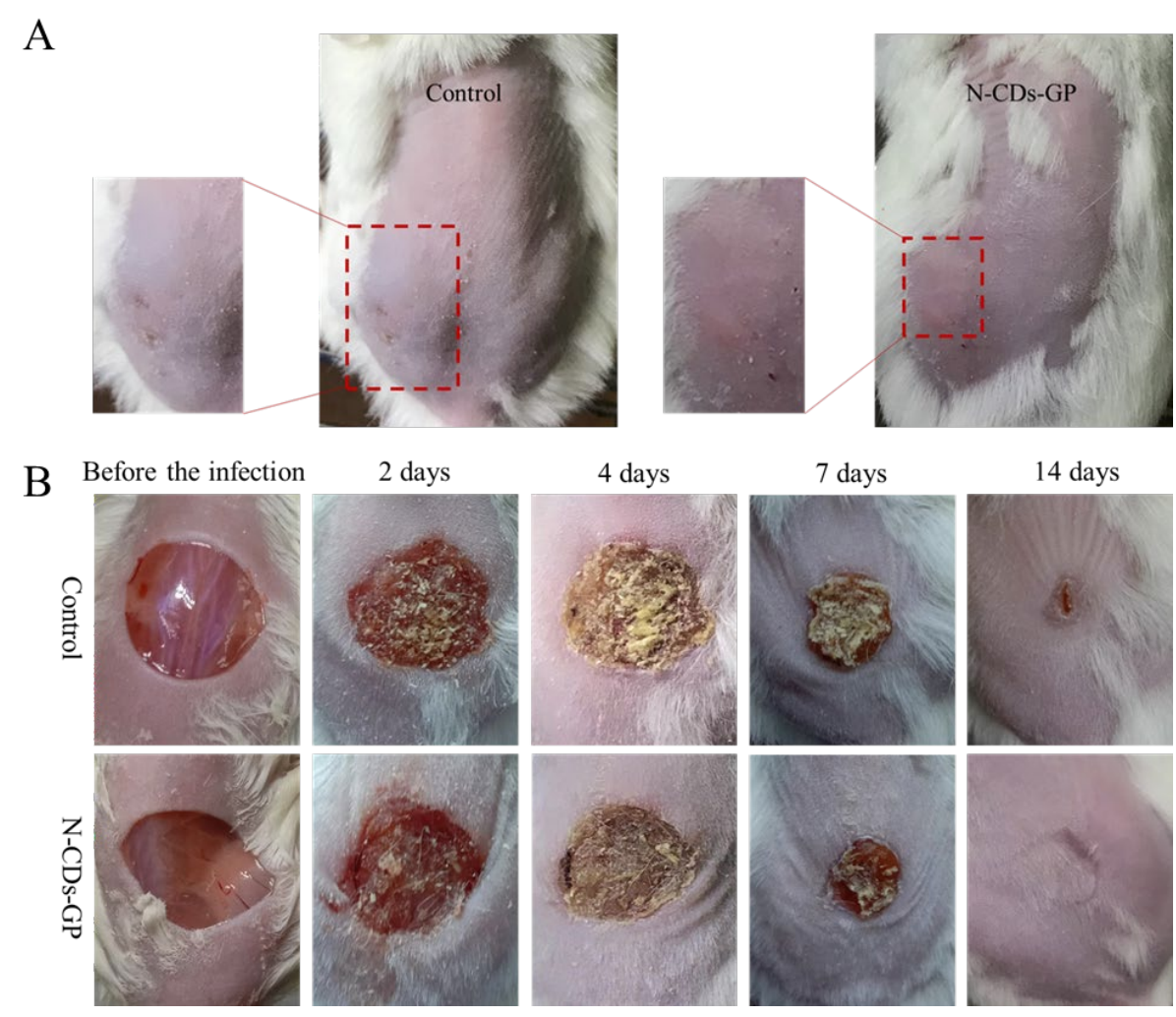

Fig. 8 In vivo antibacterial capability of N-CDs-GP. (A) Photographs of mice infected with S. aureus treated with PBS (control) and N-CDs-GP solution. (B) Photographs of the healing process of wounds infected with $S$. aureus.

\section{Conclusions}

We developed a feasible and environmentally friendly means to synthesize fluorescent nitrogen co-doped carbon dots via hydrothermal reactions and 
subsequently crosslinked the obtained N-CDs using GP. The prepared N-CDs-GP exhibited multicolor fluorescence emission, excitation wavelength-dependent properties, excellent thermal stability, broad-spectrum antibacterial ability, and excellent biocompatibility. Due to a dependence on excitation wavelengths, these N-CDs-GP nanoparticles have potential applications in cell imaging. Additionally, N-CDs-GP with strong fluorescence emission can selectively interact with Gram-positive bacteria and effectively distinguish between Gram-positive bacteria and Gram-negative bacteria. Compared with other metal-based antibiotic nanomaterials, metal-free N-CDs-GP has advantages of low preparation costs, low toxicity, and high biocompatibility. These N-CDs-GP nanoparticles can be used for cell imaging, selectively imaging Gram-positive bacteria, and effectively inhibiting bacterial growth; these particles promote the use of carbon dots for combating bacterial infections and overcoming bacterial resistance.

\section{Credit author statements}

Zhou Ninglin: Conceptualization, Methodology, Software, Funding acquisition. Shen Jian: Conceptualization, Methodology, Software, Funding acquisition. Chu Xiaohong: Validation, Formal analysis, Investigation, Data Curation, Writing-Original Draft, Writing-Review\&Editing, Visualization. Wu Fan: Formal analysis, Writing-Review\&Editing. Sun Baohong: Visualization. Zhang Ming: Conceptualization, Visualization. Song Saijie: Resources. Zhang Pan: Investigation. Wang Yuli: Validation. Zhang Qicheng: Validation.

\section{Acknowledgments}

This project was supported by the Jiangsu six category outstanding talent (2012-NY-031), Jiangsu province science and technology support plan (BE2015367), Jiangsu Science and Technology Plan (BE2019391), Jiangsu Collaborative Innovation Center of Biomedical Functional Materials.

\section{Conflicts of interest}

There are no conflicts of interest to declare. 


\section{References}

[1] J.J. Yang, X.D. Zhang, Y.H. Ma, G. Gao, X.K. Chen, H.R. Jia, Y.H. Li, Z. Chen, F.G. Wu, Carbon dot-based platform for simultaneous bacterial distinguishment and antibacterial applications, ACS Appl. Mater. Interfaces 8 (2016) 32170-32181.

[2] E.D. Brown, G.D. Wright, Antibacterial drug discovery in the resistance era, Nature 529 (2016) 336.

[3] I.M. Gould, A.M. Bal, New antibiotic agents in the pipeline and how they can help overcome microbial resistance, Virulence 4 (2013) 185-191.

[4] L.L. Li, J.H. Xu, G.B. Qi, X.Z. Zhao, F.Q. Yu, H. Wang, Core-shell supramolecular gelatin nanoparticles for adaptive and "on-demand" antibiotic delivery, ACS Nano 8 (2014) 4975-4983.

[5] G.B. Qi, L.L. Li, F.Q. Yu, H. Wang, Vancomycin-modified mesoporous silica nanoparticles for selective recognition and killing of pathogenic gram-positive bacteria over macrophage-like cells, ACS Appl. Mater. Interfaces 5 (2013) 10874-10881.

[6] D. Zhong, Y. Zhuo, Y.J. Feng, X.M. Yang, Employing carbon dots modified with vancomycin for assaying Gram-positive bacteria like Staphylococcus aureus, Biosens. Bioelectron. 74 (2015) 546-553.

[7] F.F. Xiao, B. Cao, C.Y. Wang, X.J. Guo, M.G. Li, D. Xing, X.L. Hu, Pathogen-Specific Polymeric Antimicrobials with Significant Membrane Disruption and Enhanced Photodynamic Damage To Inhibit Highly Opportunistic Bacteria, ACS Nano 13 (2019) 1511-1525.

[8] Y.W. Zhu, C. Xu, N. Zhang, X.K. Ding, B.R. Yu, F.J. Xu, Polycationic Synergistic Antibacterial Agents with Multiple Functional Components for Efficient Anti-Infective Therapy, Adv. Funct. Mater. 28 (2018) 1706709.

[9] M.I. Setyawati, C.Y. Tay, S.L. Chia, S.L. Goh, W. Fang, M.J. Neo, H.C. Chong, S.M. Tan, S.C.J. Loo, K.W. Ng, J.P. Xie, C.N. Ong, N.S. Tan, D.T. Leong, Titanium dioxide nanomaterials cause endothelial cell leakiness by disrupting the homophilic interaction of VE-cadherin, Nat. Commun. 4 (2013) 1673.

[10] K. Kaviyarasu, N. Geetha, K. Kanimozhi, C. Maria Magdalane, S. Sivaranjani, 
A. Ayeshamariam, J. Kennedy, M. Maaza, In vitro cytotoxicity effect and antibacterial performance of human lung epithelial cells A549 activity of zinc oxide doped $\mathrm{TiO}_{2}$ nanocrystals: investigation of bio-medical application by chemical method, Mater. Sci. Eng. C 74 (2017) 325-333.

[11] M.C. Wu, B.H. Ma, T.Z. Pan, S.S. Chen, J.Q. Sun, Silver-nanoparticle-colored cotton fabrics with tunable colors and durable antibacterial and self-healing superhydrophobic properties, Adv. Funct. Mater. 26 (2016) 569-576.

[12] S.F. Chen, J.P. Li, K. Qian, W.P. Xu, Y. Lu, W.X. Huang, S.H. Yu, Large scale photochemical synthesis of $\mathrm{M} @ \mathrm{TiO}_{2}$ nanocomposites $(\mathrm{M}=\mathrm{Ag}, \mathrm{Pd}, \mathrm{Au}, \mathrm{Pt})$ and their optical properties, $\mathrm{CO}$ oxidation performance, and antibacterial effect, Nano Res. 3 (2010) 244-255.

[13] M. Maruthapandi, A.P. Nagvenkar, I. Perelshtein, A. Gedanken, Carbon-Dot Initiated Synthesis of Polypyrrole and Polypyrrole@ CuO Micro/Nanoparticles with Enhanced Antibacterial Activity, ACS Appl. Polym. Mater. 1 (2019) 1181-1186.

[14] A. Sirelkhatim, S. Mahmud, A. Seeni, N.H.M. Kaus, L.C. Ann, S.K.M. Bakhori, H. Hasan, D. Mohamad, Review on zinc oxide nanoparticles: antibacterial activity and toxicity mechanism, Nano-Micro Lett. 7 (2015) 219-242.

[15] H.T. Li, Z.H. Kang, Y. Liu, S.T. Lee, Carbon nanodots: synthesis, properties and applications, J. Mater. Chem. 22 (2012) 24230-24253.

[16] C.Q. Ding, A. Zhu, Y. Tian, Functional surface engineering of C-dots for fluorescent biosensing and in vivo bioimaging, Acc. Chem. Res. 47 (2014) 20-30.

[17] H. Wang, J. Shen, Y.Y. Li, Z.Y. Wei, G.X. Cao, Z. Gai, K.L. Hong, P. Banerjee, S.Q. Zhou, Magnetic iron oxide-fluorescent carbon dots integrated nanoparticles for dual-modal imaging, near-infrared light-responsive drug carrier and photothermal therapy, Biomater. Sci. 2 (2014) 915-923.

[18] J. Liu, Y. Liu, N.Y. Liu, Y.Z. Han, X. Zhang, H. Huang, Y. Lifshitz, S.T. Lee, J. Zhong, Z.H. Kang, Metal-free efficient photocatalyst for stable visible water splitting via a two-electron pathway, Science 347 (2015) 970-974.

[19] Y.Z. Han, H. Huang, H.C. Zhang, Y. Liu, X. Han, R.H. Liu, H.T. Li, Z.H. Kang, Carbon quantum dots with photoenhanced hydrogen-bond catalytic activity in aldol 
condensations, ACS Catal. 4 (2014) 781-787.

[20] H.T. Li, R.H. Liu, W.Q. Kong, J. Liu, Y. Liu, L. Zhou, X. Zhang, S.T. Lee, Z.H. Kang, Carbon quantum dots with photo-generated proton property as efficient visible light controlled acid catalyst, Nanoscale 6 (2014) 867-873.

[21] S.J. Zhao, M.H. Lan, X.Y. Zhu, H.T. Xue, T.W. Ng, X.M. Meng, C.S. Lee, P.F. Wang, W.J. Zhang, Green synthesis of bifunctional fluorescent carbon dots from garlic for cellular imaging and free radical scavenging, ACS Appl. Mater. Interfaces 7 (2015) 17054-17060.

[22] H.Y. Yang, Y.L. Liu, Z.Y. Guo, B.F. Lei, J.L. Zhuang, X.J. Zhang, Z.M. Liu, C.F. $\mathrm{Hu}$, Hydrophobic carbon dots with blue dispersed emission and red aggregation-induced emission, Nat. Commun. 10 (2019) 1789.

[23] Y.B. Li, G.X. Bai, S.J. Zeng, J.H. Hao, Theranostic Carbon Dots with Innovative NIR-II Emission for in Vivo Renal-Excreted Optical Imaging and Photothermal Therapy, ACS Appl. Mater. Interfaces 11 (2019) 4737-4744.

[24] P. Yuan, M. Zhang, N.L. Zhou, C. Chi, X.H. Chu, B.H. Sun, S.Y. Feng, N-Doped CDs-GP nanospheres as a drug delivery nanocarrier system with carbon dots and a fluorescent tracer, New J. Chem. 41 (2017) 10880-10889.

[25] M. Zhang, W.T. Wang, F. Wu, K. Graveran, J. Zhang, C.Z. Wu, Black Phosphorus Quantum Dots Gated, Carbon-Coated $\mathrm{Fe}_{3} \mathrm{O}_{4}$ Nanocapsules (BPQDs@, ss-Fe3O4@ C) with Low Premature Release Could Enable Imaging-Guided Cancer Combination Therapy, Chem.-Eur. J. 24 (2018) 12890-12901.

[26] M. Zhang, W.T. Wang, P. Yuan, C. Chi, J. Zhang, N.L. Zhou, Synthesis of lanthanum doped carbon dots for detection of mercury ion, multi-color imaging of cells and tissue, and bacteriostasis, Chem. Eng. J. 330 (2017) 1137-1147.

[27] S. Han, H. Zhang, Y.J. Xie, L.L. Liu, C.F. Shan, X.K. Li, W.S. Liu, Y. Tang, Application of cow milk-derived carbon dots/Ag NPs composite as the antibacterial agent, Appl. Surf. Sci. 328 (2015) 368-373.

[28] H. Li, J. Huang, Y.X. Song, M.L. Zhang, H.B. Wang, F. Lu, H. Huang, Y. Liu, X. Dai, Z.L. Gu, Z.X. Yang, R.H. Zhou, Z.H. Kang, Degradable carbon dots with broad-spectrum antibacterial activity, ACS Appl. Mater. Interfaces 10 (2018) 
26936-26946.

[29] S.R. Anand, A. Bhati, D. Saini, Gunture, N. Chauhan, P. Khare, S.K. Sonkar, Antibacterial Nitrogen-doped Carbon Dots as a Reversible "Fluorescent Nanoswitch" and Fluorescent Ink, ACS Omega 4 (2019) 1581-1591.

[30] R. Jijie, A. Barras, J. Bouckaert, N. Dumitrascu, S. Szunerites, R. Boukherroub, Enhanced antibacterial activity of carbon dots functionalized with ampicillin combined with visible light triggered photodynamic effects, Colloids Surf., B 170 (2018) 347-354.

[31] R. Jijie, A. Barras, J. Bouckaert, N. Dumitrascu, S. Szunerits, R. Boukherroub, Enhanced antibacterial activity of carbon dots functionalized with ampicillin combined with visible light triggered photodynamic effects, Colloids Surf., B 170 (2018) 347-354.

[32] W.J. Lu, Y. Jiao, Y.F. Gao, J. Qiao, M. Mozneb, S.M. Shuang, C. Dong, C.Z. Li, Bright yellow fluorescent carbon dots as a multifunctional sensing platform for the label-free detection of fluoroquinolones and histidine, ACS Appl. Mater. Interfaces 10 (2018) 42915-42924.

[33] S. Li, Z. Guo, G.D. Zeng, Y. Zhang, W. Xue, Z.H. Liu, Polyethylenimine-modified fluorescent carbon dots as vaccine delivery system for intranasal immunization, ACS Biomater. Sci. Eng. 4 (2017) 142-150.

[34] X.P. Yang, M.X. Liu, Y.R. Yin, F.L. Tang, H. Hua, X.J. Liao, Green, hydrothermal synthesis of fluorescent carbon nanodots from gardenia, enabling the detection of metronidazole in pharmaceuticals and rabbit plasma, Sensors 18 (2018) 964.

[35] Y.W. Zhou, K. Hu, Z.B. Guo, K. Fang, X. Wang, F. Yang, Ning. Gu, PLLA microcapsules combined with silver nanoparticles and chlorhexidine acetate showing improved antibacterial effect, Mater. Sci. Eng., C 78 (2017) 349-353.

[36] S.K. Jou, N.A.N.N. Malek, Characterization and antibacterial activity of chlorhexidine loaded silver-kaolinite, Appl. Clay Sci. 127 (2016) 1-9.

[37] K. Saha, B.S. Butola, M. Joshi, Synthesis and characterization of chlorhexidine acetate drug-montmorillonite intercalates for antibacterial applications, Appl. Clay 
Sci. 101 (2014) 477-483.

[38] S. Holešová, M. Valášková, D. Hlaváč, J. Madejová, M. Samlíková, J. Tokarský, E. Pazdziora, Antibacterial kaolinite/urea/chlorhexidine nanocomposites: Experiment and molecular modelling, Appl. Surf. Sci. 305 (2014) 783-791.

[39] J.K. Xu, S. Strandman, J.X.X. Zhu, J. Barralet, M. Cerruti, Genipin-crosslinked catechol-chitosan mucoadhesive hydrogels for buccal drug delivery, Biomaterials 37 (2015) 395-404.

[40] A.M. Heimbuck, T. Priddy-Arrington, M.L. Padgett, C.B. Llamas, H.H. Barnett, B.A. Bunnell, M. Caldorera-Moore, Development of Responsive Chitosan-Genipin Hydrogels for Treatment of Wounds, ACS Appl. Bio Mater. 2 (2019) 2879-2888.

[41] R.A.A. Muzzarelli, M.E. Mehtedi, C. Bottegoni, A. Aquili, A.Gigante, Genipin-crosslinked chitosan gels and scaffolds for tissue engineering and regeneration of cartilage and bone, Mar. Drugs 13 (2015) 7314-7338.

[42] T. Zuo, W.M. Xu, H.L. Li, H. Song, M.H. Zhu, Geniposide and geniposidic acid, modified forms of genipin, attenuate genipin-induced mitochondrial apoptosis without altering the anti-inflammatory ability in KGN cell line, Med. Chem. Res. 26 (2017) 499-508.

[43] E. Mahgoub, S.M. Kumaraswamy, K.H. Kader, B. Venkataraman, S. Ojha, E. Adeghate, M. Rajesh, Genipin attenuates cisplatin-induced nephrotoxicity by counteracting oxidative stress, inflammation, and apoptosis, Biomed. Pharmacother. 93 (2017) 1083-1097.

[44] K.N. Nam, Y.S. Choi, H.J. Jung, G.H. Park, J.M. Park, S.K. Moon, K.H. Cho, C. Kang, I. Kang, E.H. Lee, Genipin inhibits the inflammatory response of rat brain microglial cells, Int. Immunopharmacol. 10 (2010) 493-499.

[45] K. Okada, J. Shoda, M. Kano, S. Suzuki, N. Ohtake, M. Yamamoto, H. Takahashi, H. Utsunomiya, K. Oda, K. Sato, A. Watanabe, T. Ishii, K. Itoh, M. Yamamoto, T. Yokoi, K. Yoshizato, Y. Sugiyama, H. Suzuki, Inchinkoto, a herbal medicine, and its ingredients dually exert Mrp2/MRP2-mediated choleresis and Nrf2-mediated antioxidative action in rat livers, Am J Physiol Gastrointest Liver Physiol, 292 (2007) G1450-G1463. 
[46] S. Zhang, X.B. Pei, Y.Y. Xue, J.Y. Xiong, J. Wang, Bio-safety assessment of carbon quantum dots, N-doped and folic acidmodified carbon quantum dots: A systemic comparison, Chin. Chem. Lett. (2019).

[47] Y. Yang, X.L. Ren, Z.N. Sun, C.H. Fu, T.L. Liu, X.W. Meng, Z.L. Wang, Toxicity and bio-distribution of carbon dots after single inhalation exposure in vivo, Chin. Chem. Lett. 29 (2018) 895-898.

[48] F. Wu, M. Zhang, X.H. Chu, Q.C. Zhang, Y.T. Su, B.H. Sun, T.Y. Lu, N.L. Zhou, J. Zhang, J.X. Wang, X.Y. Yi, Black phosphorus nanosheets-based nanocarriers for enhancing chemotherapy drug sensitiveness via depleting mutant p53 and resistant cancer multimodal therapy, Chem. Eng. J. 370 (2019) 387-399.

[49] F. Wu, B.H. Sun, X.H. Chu, Q.C. Zhang, Z.C. She, S.J. Song, N.L. Zhou, J. Zhang, X.Y. Yi, D.H. Wu, J.X. Wang, Hyaluronic Acid-Modified Porous Carbon Coated $\mathrm{Fe}_{3} \mathrm{O}_{4}$ Nanoparticles for Magnetic Resonance Imaging-Guided Photothermal/Chemo-Therapy of Tumor, Langmuir 35 (2019) 13135-13144.

[50] F. Lu, Y.X. Song, H. Huang, Y. Liu, Y.J. Fu, J. Huang, H. Li, H.H. Qu, Z.H. Kang, Fluorescent carbon dots with tunable negative charges for bio-imaging in bacterial viability assessment, Carbon 120 (2017) 95-102.

[51] M. Beeby, J.C. Gumbart, B. Roux, G.J. Jensen, Architecture and assembly of the Gram-positive cell wall, Mol. Microbiol. 88 (2013) 664-672.

[52] O. Abdi, M. Kazemi, A review study of biosorption of heavy metals and comparison between different biosorbents, J. Mater. Environ. Sci. 6 (2015) 1386-1399.

[53] A. Mai-Prochnow, M. Clauson, J. Hong, A.B. Murphy, Gram positive and Gram negative bacteria differ in their sensitivity to cold plasma, Sci. Rep. 6 (2016) 38610. [54] D.A. Dik, J.F. Fisher, S. Mobashery, Cell-wall recycling of the Gram-negative bacteria and the nexus to antibiotic resistance, Chem. Rev. 118 (2018) 5952-5984.

[55] O. Jeske, M. Schüler, P. Schumann, A. Schneider, C. Boedeker, M. Jogler, D. Bollschweiler, M. Rohde, C. Mayer, H. Engelhardt, S. Spring, C.Jogler, Planctomycetes do possess a peptidoglycan cell wall, Nat. Commun. 6 (2015) 7116.

[56] A. Espaillat, O. Forsmo, K. El Biari, R. Björk, B. Lemaitre, J. Trygg, F.J. 
Cañada, M.A. Pedro, F. Cava, Chemometric analysis of bacterial peptidoglycan reveals atypical modifications that empower the cell wall against predatory enzymes and fly innate immunity, J. Am. Chem. Soc. 138 (2016) 9193-9204.

[57] N.A. Travlou, M. Algarra, C. Alcoholado, M. Cifuentes-Rueda, A.M. Labella, J. M. Lázaro-Martínez, E. Rodríguez-Castellón, T.J. Bandosz, Carbon quantum dot surface-chemistry-dependent Ag release governs the high antibacterial activity of Ag-metal-organic framework composites, ACS Appl. Bio Mater. 1 (2018) 693-707.

[58] P. Yadav, S.T. Nishanthi, B. Purohit, A. Shanavas, K. Kailasam, Metal-free visible light photocatalytic carbon nitride quantum dots as efficient antibacterial agents: An insight study, Carbon 152 (2019) 587-597.

[59] J. Tang, Y. Song, S. Tanvir, W.A. Anderson, R.M. Berry, K.C. Tam, Polyrhodanine coated cellulose nanocrystals: a sustainable antimicrobial agent, ACS Sustainable Chem. Eng. 3 (2015) 1801-1809. 